\title{
Begleitung von Frauen mit postpartalen psychischen Erkrankungen
}

\section{Anne Wiecker}

Viele Mütter entwickeln in den ersten Tagen nach der Geburt ein Stimmungstief. Geprägt von Traurigkeit und Schuldgefühlen hüllt es diese vermeintlich so glückliche erste Zeit mit dem Baby in graue Wolken. Dauert die trübe Stimmung lange an oder tritt sie wiederholt auf, könnte eine postpartale Depression (PPD) vorliegen. Hebamme Anne Wiecker hat viele Frauen mit psychischen Erkrankungen nach der Geburt begleitet. Mit praktikablen Tipps und einem Fallbeispiel erklärt sie, was für die Wochenbettbetreuung dabei wichtig ist.

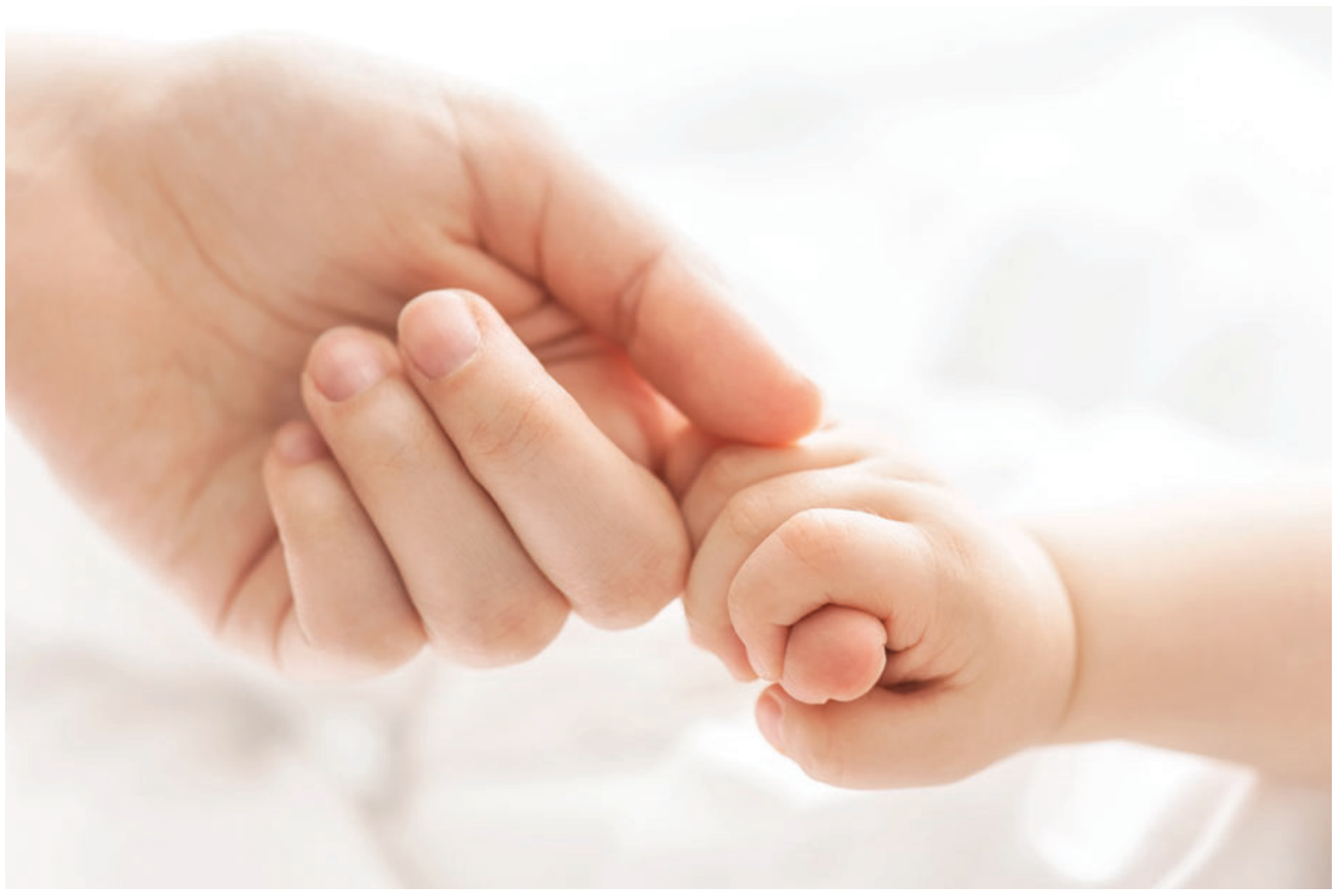

Abb. 1 Treten im Wochenbett depressive Verstimmungen der Mutter auf, können diese die Mutter und die Bindung zum Kind beeinflussen. Die Hebamme kann der Frau in der Krise eine bedeutsame Hilfe zur Selbsthilfe sein. (Foto: Alexandr Vasilyev stock.adobe.com)

Die Rolle der Hebamme bei einer

\section{Wochenbettdepression}

Symptome für eine Wochenbettdepression bei der Frau können schwerwiegende Folgen für die ganze Familie nach sich ziehen. In dieser empfindsamen Zeit der Schwangerschaft, Geburt und des Wochenbetts ist keiner Berufsgruppe so ein tiefer Einblick in das Befinden der Frau möglich wie uns Hebammen. Wir begleiten Frauen und Männer, wenn die Geburt ihres Kindes sie zu einer Familie 
macht. Und wir haben hier die Möglichkeit, gezielt Hilfestellung zu bieten. Aus diesem Privileg erwächst gleichsam eine Pflicht: Es gilt, depressive Episoden zu erkennen, zu begleiten und eine interdisziplinäre Zusammenarbeit zu generieren, um die Familie zu schützen.

In meiner Hebammentätigkeit erlebe ich eine deutliche Zunahme psychischer Vorerkrankungen und Depressionen nach der Geburt. Die Gründe dafür sind aus meiner Sicht vielschichtig. So führte u.a. eine vermehrte Aufklärung in den vergangenen Jahren dazu, dass dieses Thema medial bearbeitet wird. Prominente erzählen öffentlich von ihren Depressionen und Printmedien enthalten immer häufiger Artikel zu diesem Thema. Die Depression wird sozusagen gesellschaftsfähig. Die Aufklärung trägt positiver Weise dazu bei, dass sie als ernstzunehmende Krankheit wahrgenommen wird. Gleichzeitig ist zu beobachten, dass ein enormer gesellschaftlicher Druck auf Müttern und jungen Familien lastet.

Häufig erlebe ich Frauen, die zweifeln, ob sie die mit der Mutterschaft verbundenen Erwartungen und Anforderungen erfüllen können. Der persönliche Leistungsdruck, einem gesellschaftlichen Idealbild zu entsprechen, ist nie höher gewesen als heute. Gleichzeitig fällt vielfach der familiäre Rückhalt weg, den in früheren Zeiten etwa die Großeltern boten. Familien wohnen oft nur im engsten Kreis beieinander und die komplette Verantwortung für die Kinder und deren Erziehung wird bei den Eltern gesehen. In einer Gesellschaft, in der Leistungsbereitschaft die höchste Maxime darstellt, liegen die Erwartungen an eine Mutter sehr hoch. Viele Mütter, die ich als Beleghebamme betreue, haben zusätzlich psychische Vorerkrankungen.

Es stellt sich die Frage, wie wir Hebammen diese Frauen und Familien an dieser Stelle unterstützen können: Welche Frühwarnsysteme und welche Möglichkeiten der Hilfestellung können wir nutzen?

\section{Sorgfältige Anamnese und engmaschige Betreuung}

Postpartale Depressionen sind aufgrund des schleichenden Verlaufs meist schwierig zu erkennen. Die Symptome einer PPD, z. B. das Gefühl von Wertlosigkeit und Interessenlosigkeit [7][3], treten oft erst nach Verlassen des Krankenhauses auf. In den folgenden Wochen sehen die Frauen den Kinderarzt oft nur einmal und den ambulanten Gynäkologen nur noch zur Abschlussuntersuchung. Das birgt ein gewisses Risiko zu übersehen, dass eine Frau eine PPD entwickelt hat, wenn sie die Veränderung nicht direkt selbst anspricht. Ich beobachte, dass betroffene Frauen oft ihre Gefühle zu verschweigen versuchen, um in ihrer Mutterrolle zu funktionieren. Wenn ihnen die Traurigkeit bewusst ist, empfinden sie ein großes Scham- und Schuldgefühl oder es machen sich Versagensängste breit. Das soziale Umfeld bemerkt Veränderungen der
Wöchnerin häufig nicht. Und dem Partner fällt es schwer, seine Beobachtungen in Worte zu fassen. Häufig gelingt das erst, wenn ich ihn direkt auf Veränderungen bei seiner Frau anspreche.

Hier haben wir Hebammen eine Ressource: Wir sehen die Frauen während der Wochenbettbetreuung sehr regelmäßig und können die Zeit nutzen, um neben körperlichen Veränderungen die Emotionen der Frau zu verbalisieren. Bei Frauen, die ich schon in der Schwangerschaft betreuen konnte, habe ich bis zum Wochenbett ein Gefühl für die Person entwickelt. Dies ist besonders hilfreich, um schon kleine Veränderungen der psychischen Verfassung zu bemerken. Im Idealfall versuche ich, eine engmaschige Betreuung besonders im ersten Monat nach der Geburt zu gewährleisten.

Merke
Für Hebammen ist es wichtig, sich ausreichend Zeit für
eine ausführliche Anamnese zu nehmen, um klassische
Risikofaktoren für eine PPD zu analysieren. Vor allem
eigene und familiäre psychische Vorerkrankungen oder
ein erlebtes Trauma können statistisch häufiger zu
einer PPD führen [7][3].

\section{Die Frau und den Partner aufklären}

Das Mutterwerden wird in der heutigen Zeit mehr denn je zu einem besonderen Ereignis. Viele Paare zelebrieren die Schwangerschaft. In Verbindung mit dem wachsenden Hebammenmangel hat das zur Folge, dass Frauen sich heute oft schon sehr früh um Kontakt zu einer Hebamme bemühen. Daraus ergibt sich die Chance einer langfristigen individuellen Hebammenbetreuung.

Für eine umfassende Beratung in der Schwangerschaft habe ich mir einen Leitfaden mit wichtigen Themen rund um Schwangerschaft, Geburt und Wochenbett erstellt (vgl. [1]). Neben den körperlichen Prozessen im Wochenbett skizziere ich den Schwangeren auch die psychischen Veränderungen, die sie erwarten könnten. Dabei ist es mir wichtig, keine Ängste zu schüren, sondern sachlich zu informieren. Jeder Frau biete ich im Beratungstermin Informationen darüber an, was Babyblues, Wochenbettdepression und Wochenbettpsychose bedeuten und mit welcher Wahrscheinlichkeit Frauen davon betroffen sind. Ich versuche die Schwangere für dieses Thema zu sensibilisieren und gebe Hinweise, woran die Frauen selbst erkennen können, dass sie sich in einer Depression befinden.

Darüber hinaus gestalte ich zwei Stunden meines Geburtsvorbereitungskurses zum Thema Wochenbett. Zu diesem Kursteil empfehle ich allen Frauen, ihren Partner mitzubringen. Ich ermutige die Männer, auf ihre Frauen achtzugeben und beobachtete psychische Veränderungen ihrer Frauen zum Wohle der ganzen Familie offen anzusprechen. 
Seitdem ich für das Thema schon in der Schwangerschaft sensibilisiere, sind es in der Wochenbettbetreuung eher die Partner, die das Gespräch zu mir suchen, weil ihnen depressive Veränderungen an ihrer Partnerin auffallen.

\section{Merke}

Die Einbeziehung des Partners stellt einen elementaren Faktor in der Früherkennung einer PPD dar.

Zum Abschluss des Geburtsvorbereitungskurses bekommen die Frauen von mir ein Handout, in dem gebündelte Informationen zu allen besprochenen Themen nachzulesen sind. Hier sind u. a. Kontaktadressen zu Notfallseelsorger, Selbsthilfegruppen und Psychologen vermerkt, damit auch Frauen, die ich nicht im Wochenbett betreue, einen Notfallplan haben.

Ergeben sich in der Anamnese bei der Schwangeren Symptome für eine psychische Erkrankung, nutze ich die deutschsprachige Version des Edingburgh Postnatal Depression Scale (EPDS) schon in der Vorsorge als Gesprächsleitfaden, um ggf. depressive Verstimmungen möglichst früh zu erkennen. Damit spreche ich Fragen an, wie z. B.: „Sind Sie in letzter Zeit oft gereizt oder wütend?“ - „Haben Sie negative Gefühle im Hinblick auf die Schwangerschaft oder das Baby?“ - „Weinen Sie oft und fühlen sich unglücklich?“ In der Gesprächssituation vermittele ich der Frau, sich auch mit negativen Gefühlen öffnen zu können. Je nach Ergebnis unterstütze ich die Schwangere im Erstellen eines Selbsthilfeplans bzw. in der Kontaktaufnahme mit einem Arzt oder Psychologen.

\section{Gemeinsam einen Selbsthilfeplan erstellen}

Werden in der Wochenbettbetreuung depressive Verstimmungen deutlich, ist es zunächst wichtig, herauszufinden, inwieweit diese die Mutter und die Bindung zum Kind beeinflussen. Ein individuell entworfener Selbsthilfeplan kann helfen, die Bedürfnisse der Beteiligten zu visualisieren und somit der Frau Verständnis und Entlastung zukommen zu lassen. Hierbei ist es mir wichtig, die Frau in der Ideenfindung zu unterstützen. Selbst entwickelte Ideen lassen sich oft besser und schneller umsetzen als vorgegebene Ziele. Hierbei bietet das Buch „Psychologie und Pathopsychologie“ eine gute Vorlage [2].

Zusätzlich suche ich mit der betroffenen Frau nach einem Notfallkontakt. Dies sollte eine enge Vertrauensperson sein, der es möglich ist, rund um die Uhr für die Frau erreichbar zu sein. In Situationen, die sie zu überwältigen drohen, ist es oft hilfreich, wenn die Möglichkeit besteht, sich durch vertraute Menschen Unterstützung einzuholen.

Auch wenn ich dieses Instrument oft sehr erfolgreich einsetzen konnte, ist es wichtig, die Grenzen der Selbsthilfe rechtzeitig zu erkennen: 
Merke

Bessert sich die Situation der Frau innerhalb weniger

Tage nicht oder Verschlimmern sich die Symptome, ist die Frau nicht gewillt oder in der Lage die selbst erarbeiteten Schritte umzusetzen oder kommen suizidale Gedanken hinzu, ist ein schnelles Reagieren seitens der Hebamme wichtig und eine professionelle Unterstützung unabdingbar.

\section{Mutter-Kind-Bindung und -Interaktion fördern}

Aus einer PPD resultiert in der Regel eine Beziehungsstörung zwischen Mutter und Kind. Die Mütter ziehen sich zurück, reden wenig bis gar nicht mit dem Säugling und zeigen kaum körperliche Zuwendung. Dazu zeigen sie eine geringe Sensitivität für die kindlichen Signale. Neugeborene von depressiven Müttern zeigen oft ein gereiztes Verhalten, weinen häufig und heftiger, können sich nur schlecht selbst regulieren oder wirken passiv. Es gilt, diese Kaskade zu unterbrechen, da eine lang anhaltende gestörte Interaktion bei Nichtbehandlung der Mutter zu Entwicklungsstörungen bis hin zu psychischen Erkrankungen des Kindes führen kann [6][7].

Die Unterstützung in der Mutter-Kind-Interaktion habe ich bei meinen bisherigen Fällen von Wochenbettdepressionen als wichtigsten, grundlegenden Baustein einer erfolgreichen Therapie erlebt. Ein sehr einfaches und schnell anzuwendendes Instrument zum Bindungsaufbau ist die Unterstützung des direkten Körperkontakts zwischen Mutter und Kind. Besonders bei Frauen, die eine traumatische Geburt als Auslöser für die PPD zeigen, ist ein Bondingbad [5] sinnvoll. Der enge Körperkontakt hierbei und die oft liebevoll gestaltete Umgebung des Bads sorgen für einen sehr innigen und privaten Moment. Oft habe ich erlebt, dass Frauen sich bei diesen Bädern öffnen und ihre Ängste formulieren können. In Verbindung mit (angeleiteter) Babymassage ist eine intensive körperliche Nähe zu erwarten. Hier ist es wichtig, der Frau mit maximalem Respekt und einer hohen Wertschätzung gegenüber ihrer Leistung als Mutter zu begegnen und auch Tränen Raum zu geben.

\section{Die Mutter beim Stillen unterstützen}

Darüber hinaus sollte eine Frau, die während einer PPD stillen möchte, unbedingt in diesem Vorhaben unterstützt werden. Neben den zahlreichen positiven Faktoren der Muttermilch ist kaum eine andere Begegnung zwischen Mutter und Kind inniger. Hier erlebt die Mutter deutlich, dass sie eine elementare Rolle für ihr Kind spielt. Viele Mütter mit einer PPD leiden unter Insuffizienzgefühlen. Sie denken, dass sie nicht ausreichend für ihr Kind sind und dass sie dieses nicht adäquat versorgen können.
Merke

Durch Unterstützung beim Stillen und die Ermutigung zu engem Körperkontakt mit dem Säugling kann die Hebamme der Frau ohne viele Worte zeigen, wie wichtig sie als Mutter für ihr Kind ist.

\section{Zu gemeinsamen Aktivitäten motivieren}

Ist der Genesungsprozess vorangeschritten, habe ich gute Erfahrungen damit gemacht, Mutter und Kind gemeinsame Aktivitäten zu ermöglichen. So kann zum Beispiel die Teilnahme an einem DELFI-Kurs oder das Babyschwimmen die gemeinsame Zeit positiv besetzen und der Mutter die Möglichkeit geben, in Interaktion mit dem Kind zu kommen. Zusätzlich erfährt hierbei die Mutter eine Etablierung im Mütterkreis. Sie lernt andere junge Familien kennen und findet dort Verbindungen, die wiederum eine Entlastung mit sich bringen können. Hat das Kind Gefallen an solchen Aktivitäten, stärken sie zusätzlich das Selbstbewusstsein der Frau, der dabei ihre mütterlichen Fähigkeiten erneut bewusst vor Augen geführt werden.

\section{Wann professionelle Unterstützung wichtig ist}

Mit Hilfe der EPDS kann die Hebamme die depressiven Symptome der Frau und die Bedeutung von professioneller Begleitung verbalisieren. Bei einem Wert ab 13 sollte die Frau darin unterstützt werden, zur weiteren Abklärung einen Arzt aufzusuchen (Marcé Gesellschaft für Peripartale Psychische Erkrankungen e.V.). Entwickelt sich eine akute psychische Krise, ist eine professionelle Unterstützung elementar. Hebammen können hier interdisziplinäre Strukturen schaffen, um der Familie möglichst schnell helfen zu können. Nachdem ich meine ersten Mütter mit akuten Depressionen betreut hatte, habe ich Kontakt zu Therapeuten und Neurologen gesucht, die im Notfall Erste Hilfe bei akut gefährdeten Frauen leisten können. Durch diese interdisziplinäre Vernetzung ist es mir inzwischen möglich, innerhalb weniger Tage einen ersten Beratungs-termin für die Frauen zu generieren und im Akutfall sogar sofort eine medikamentöse Einstellung einzuleiten. Zwar versichern die Krankenkassen einen Beratungstermin innerhalb der ersten vier Wochen nach Kontaktaufnahme mit einem Therapeuten, allerdings kann im Akutfall diese Zeit nicht überbrückt werden und die Situation kann eskalieren.

\footnotetext{
Merke

Hebammen sollten selbst in die Kommunikation mit wohnortnahen Psychologen gehen und ein Netzwerk mit behandelnden Praxen bzw. Organisationen aufbauen, um für ihre betreuten Frauen die Beratungsschwelle und die Wartezeit bis zum Erstkontakt herabzusetzen.
}

Oft gibt es in den Städten Beratungseinrichtungen, die ihrerseits mit Psychologen zusammenarbeiten und 
schnell und unbürokratisch Hilfe leisten können. So ist z.B. der Paritätische Wohlfahrtsverband eine erste Anlaufstelle für jegliche Notsituation rund um die Schwangerschaft. Das dortige Team besteht aus geprüften Notfallseelsorger/-innen und verfügt über ein gut ausgebautes Netz an psychologischen Mitarbeitern.

\section{Geeignete Klinik zur stationären Behandlung finden}

Ist eine stationäre Behandlung nötig, da die Depression z. B. eine sichere Versorgung des Kindes im Alltag nicht mehr zulässt, sollte möglichst eine Klinik gewählt werden, die das Kind mit aufnehmen kann. Das bietet die Chance, die Störung in der Mutter-Kind-Beziehung effektiv mit zu behandeln [8][9]. Leider gibt es deutschlandweit sehr wenig kombinierte Behandlungsplätze und die Wartezeit ist oft sehr lang. Ist eine akute Deeskalation wie im Fallbeispiel nötig, sollte zumindest eine nahe gelegene Frauenklinik eine Wiederaufnahme gewährleisten und das Kind dort als Begleitperson (im Idealfall auch den Partner im Rahmen eines Familienzimmers) mit aufnehmen. Lässt sich in naher Zukunft kein Mutter-Kind-Therapieplatz in einer geeigneten Einrichtung finden, ist die Therapie in einer Tagesklinik eine Option. Wichtig ist auch hier die Einbeziehung des Säuglings.

\section{Das Umfeld stabilisieren}

Neben dem therapeutischen Ansatz zur Behandlung einer Wochenbettdepression ist die Stabilisierung des Umfelds von Frau und Kind ein wichtiger Faktor. Hierbei geht es vor allem um die Entlastung der Frau und um die Etablierung einer Alltagsstruktur. Dafür ist es hilfreich, wenn der Partner während der Akutzeit der Depression an der Seite der Frau sein kann. Kurzfristig ist dies über die Beantragung von Urlaub möglich. Ratsam ist dazu die Inanspruchnahme von Elternzeit für den Partner. Die Hebamme kann mit der betroffenen Familie im nahen Umfeld der Frau nach weiteren geeigneten Vertrauenspersonen suchen. Vielleicht können die Eltern einen Teil der Hausarbeit übernehmen oder die beste Freundin kann zu festen Zeiten den Kinderwagen schieben? Sind schon größere Kinder in der Familie, sollte nach geeigneten Betreuungsmöglichkeiten gesucht werden. Oft ist ein auf sich allein gestellter Vater mit der Situation und der Fülle von Aufgaben überfordert. Neben der Hilfe von Familie und Freunden ist die Rezeptierung einer Haushaltshilfe durch die behandelnden Ärzte möglich und sollte in jedem Fall genutzt werden. Darüber hinaus kann die Hebamme mit dem Paar einen Alltagsplan erstellen. Hierbei gibt die Hebamme Unterstützung, alle anfallenden Aufgaben aufzulisten und einem Verantwortlichen zuzuordnen. Dies schafft Übersicht und vor allem Struktur. Mit zunehmendem Behandlungserfolg kann die Frau langsam immer mehr Alltagsaufgaben zurückgewinnen. 


\section{Schlussbetrachtung}

Bei der wachsenden Zahl psychischer Vorerkrankungen und dem wachsenden Druck, der auf Müttern lastet, wird die Zahl der Frauen, die an einer PPD leiden, stetig zunehmen. Daher ist es wichtig, dass besonders Hebammen, die in dieser empfindlichen Phase Zugang zu den Frauen und deren Familien haben, ein individuelles System entwickeln, welches depressive Symptome frühzeitig erkennt. Hebammen sollten die Grenzen ihrer Möglichkeiten bewusst sein und sie sollten möglichst schnell die interdisziplinäre Zusammenarbeit suchen. Gut entwickelte Netzwerke helfen, gezielte Hilfestellungen zu geben, um die Frauen optimal zu betreuen.

\section{KONTAKT}

\section{Rat und Hilfe}

- Die Stiftung Deutsche Despressionshilfe bietet kompakte Artikel rund um Depressionen in der Schwangerschaft und nach der Geburt: www.deutsche-depressionshilfe.de/depressioninfos-und-hilfe/depression-in-verschiedenen-fac etten/in-der-schwangerschaft-und-nach-dergeburt

- Schatten \& Licht e. V. ist eine 1996 von betroffenen Frauen gegründete Selbsthilfe-Organisation. Sie befasst sich u. a. mit peripartaler Depression, Angst- oder Zwangsstörung und peripartaler Psychose: www.schatten-und-licht.de

- Der Verein Postnatale Depression Schweiz stellt auf seiner Website unter der Rubrik Fachhilfe verschiedene Therapieformen zur Behandlung dieser Krankheit vor. In der Rubrik Selbsthilfe gibt es anschauliche Präventionstipps, zudem steht die Edinburgh-Postnatal-Depressions-Skala (EPDS) zum Download bereit: www.postnatale-d epression.ch

- Die Initiative Frauen Gesundheit Familien Zukunft e. V. hat sich die Prävention peripartaler psychischer Erkrankungen zum Ziel gesetzt und organisiert u. a. Schulungen für Hebammen und andere Fachpersonen, die mit Schwangeren, Gebärenden, Wöchnerinnen und jungen Müttern arbeiten. Auch Betroffene und Angehörige finden Tipps, wie sie mit Krisen umgehen, Stabilität erhalten, Hilfe annehmen und Veränderung ermöglichen können: www.fgfz-online.de

- Die Marcé Gesellschaft für Peripartale Psychische Erkrankungen e. V. ist die deutschsprachige Sektion der International Marcé Society for Psychaitric Disorders of Childbearing. Sie bietet ebenfalls Fortbildungen für Fachpersonal an und setzt sich für die Verbesserung der Versorgungsangebote und die Entstigmatisierung der Erkrankungen ein: www.marce-gesellschaft.de

\section{Fallbeispiel: PPD in der Wochenbettbetreuung}

\section{Das Kennenlerngespräch}

Ich lerne die 28-jährige Anna* (alle mit* markierten Namen sind redaktionell verändert) in der 8. SSW kennen. Sie und ihr Partner Niklas* erwarten ihr erstes gemeinsames Kind. Niklas* hat aus einer früheren Beziehung einen 5-jährigen Sohn, zu dem er und Anna* regelmäßigen und guten Kontakt haben. Leon* besucht das Paar jedes zweite Wochenende und hat in Anna* eine enge Bezugsperson gefunden. Beim Kennenlerngespräch mit dem Paar berichtet Anna* detailliert über ihre Lebenssituation: Sie arbeitet Vollzeit als Gymnasiallehrerin und unterrichtet ihre erste eigene Klasse als Klassenleiterin. Sie möchte möglichst lange in der Schwangerschaft arbeiten, um das Schuljahr mit der Klasse beenden zu können. Niklas* äußert schon im ersten Gespräch Zweifel an diesem Plan. Er arbeitet als Berufssoldat 500 km entfernt und sieht Anna nur am Wochenende. Dadurch werde er Anna* deutlich weniger unterstützen können. Er verweist darauf, dass Anna* hohe Ansprüche an sich selbst habe. Seine Zweifel wirken aufrichtig. Im weiteren Gespräch wird klar, dass Anna* einen genauen Plan von ihrer Schwangerschaft und der ersten Zeit als Mutter hat. Sie wirkt auf mich perfektionistisch und euphorisch. Sie wünscht sich eine engmaschige Betreuung von Hebammenseite und eine Beleggeburt, da es für sie nicht vorstellbar ist, jemandem Fremden in einer so sensiblen Phase Vertrauen zu schenken. Wir vereinbaren eine Hebammenbetreuung zur Vorsorge, Beleggeburt, Geburtsvorbereitung und Wochenbettbetreuung. Die Vorsorge erfolgt gemeinsam mit dem Gynäkologen.

\section{Die Betreuung in der Schwangerschaft}

In den folgenden Wochen sehe ich Anna* fast jede zweite Woche zu Einzelterminen aufgrund allgemeiner Schwangerschaftsbeschwerden und ab der 22. SSW zudem zum wöchentlichen Geburtsvorbereitungskurs. Anna* plagen viele Fragen und Sorgen. Durch aktives Zuhören und Nachfragen unterstütze ich Anna*, ihre Gefühle und Bedürfnisse zu verbalisieren und eigene Lösungen für ihre Probleme zu finden. Wichtig ist mir, dass sie in Kontakt mit ihrem Kind bleibt.

Je weiter die Schwangerschaft voranschreitet, desto mehr habe ich das Gefühl, dass Anna* wenig Vertrauen in sich und ihren Körper hat. Sie möchte jede Veränderung im Detail bereden und wird zunehmend unsicherer, was ihre Rolle als Mutter angeht. Bei jedem Termin begrüßt sie mich mit den Worten: „Ich bin so froh, dass du da bist. Ich habe die Kleine heute nicht gespürt. Kannst du die Herztöne hören?" Schon bei der palpatorischen Untersuchung spüre ich deutliche Kindsbewegungen, die Anna* selbst 
aber nicht wahrzunehmen scheint. Ich bitte sie daher, jeden Abend Kontakt mit dem Kind aufzunehmen. Sie stimmt zu und wird täglich mithilfe von Wahrnehmungsübungen in die Kommunikation mit dem Ungeborenen gehen. Schon beim nächsten Termin berichtet sie, dass sie die Kindsbewegungen jetzt deutlicher spürt, und sie wirkt beruhigter. Dennoch habe ich das Gefühl, ihre Gedanken und der Zwang zum Planen lassen sie nicht zur Ruhe kommen. Sie hat massive Schwierigkeiten, sich auf die Schwangerschaft einzulassen, da sie „nicht alles unter Kontrolle" hat. Aus diesem Grund möchte sie den Geburtsvorbereitungskurs sehr früh beginnen. Sie braucht für sich die Sicherheit, alles zu wissen und nichts Elementares zu vergessen.

In der 29. SSW meldet sich Niklas* nachts bei mir, da Anna* sehr regelmäßig und in kurzen Abständen krampfartige Bauchschmerzen hat. Ich höre sie im Hintergrund schwer atmen und bitte beide, mich in der Klinik zu treffen. In der Klinik bestätigen sich regelmäßige Kontraktionen. Die Zervix ist auf $1 \mathrm{~cm}$ verkürzt und der Muttermund ist fingerdurchgängig. Anna* erhält somit eine Bolustokolyse, Lungenreife-Behandlung und strenge Bettruhe. Unter der Tokolyse ist sie wehenfrei. Allerdings ist ihre psychische Verfassung desolat. Anna* ist am Boden zerstört, dass sie jetzt nicht mehr arbeiten kann und die Sorge um ihr Kind lässt sie nicht mehr los. Immer wieder fallen Sätze wie: „Ich wusste, dass mein Plan nicht funktioniert.“ oder „Mein Kind will einfach nicht bei mir bleiben." Während des stationären Aufenthalts war ich regelmäßig bei ihr, um auf ihre umfangreichen Sorgen und Ängste einzugehen. Niklas* war sehr hilflos und kam nach eigenen Angaben überhaupt nicht mehr an sie heran. Um Anna* professionell zu unterstützen, organisierten wir Kontakte mit der Klinikpsychologin. Anna* suchte in den Gesprächen immer wieder die Bestätigung, eine gute Mutter zu sein. Nach und nach konnte ihr Selbstbewusstsein wieder aufgebaut werden. In der 35. SSW konnte sie ins häusliche Milieu entlassen werden. Anna* freute sich die letzten Wochen mit Niklas* das Nest vorzubereiten und war stolz, die Situation gemeistert zu haben.

\section{Die Geburt und die ersten Tage pp.}

6 Tage vor dem errechneten Geburtstermin meldete sich Anna* mit regelmäßigen Wehen bei mir. 7 Stunden später brachte sie ein gesundes Mädchen zur Welt. Die Geburt war komplikationslos und ohne Interventionen. Anna* und Niklas* durch ausgiebiges Bonding mit ihrer Tochter intensiv in Kontakt treten und das erste Stillen verlief erfolgreich. Anna* wirkte nach der Geburt gelöst und unendlich Stolz auf ihre Leistung. Sie verbrachte die nächsten 3 Tage mit Niklas* und Mia* im Familienzimmer der Wochenstation und benötigte kaum Unterstützung. Beim Wochenbettbesuch im Krankenhaus erlebte ich sie gelöst und voller Elan.

\section{BUCHTIPPS}

\section{Literatur- und Surftipps für Eltern}

- Anke Rohde: Postnatale Depressionen und andere psychische Probleme - ein Ratgeber für betroffene Frauen und Angehörige. Kohlhammer, 2014

- Tanja Sahib: Darauf waren wir nicht vorbereitet. Books on Demand, 2018

- Stephanie Herrmann: Postpartale Depressionen Wege zu einer sicheren Mutter-Kind-Bindung, Diplomica-Verlag, 2014

- Petra Wiegers: Nur die Liebe fehlt - von Depressionen nach der Geburt und Müttern, die ihr Glück erst finden müssen. Patmos, 2016

- Stefanie Bunjes: Tränen, Liebe, Freiheitsdrang Mein schwerer Start ins Mutterdasein. Independently published, 2019

- Techniker Krankenkasse, Landesvertretung Bayern (Hg.): Depression nach der Geburt - Ratgeber für Betroffene und Angehörige. Iv-bayern@tk.de, München 2014

" www.gesundheitsinformation.de - Depression nach der Geburt - was kann helfen?

\section{Der Wochenbettverlauf}

Am 4. Tag postpartum besuchte ich die Familie zu Hause. Anna* berichtete mir von einer eher unruhigen Nacht. Mia* hätte ganz gut geschlafen, sie selbst sei aber irgendwie nicht zur Ruhe gekommen. Sie öffnete sich und berichtete von ihren Ängsten, dass Mia* etwas passieren würde und sie dann nicht richtig würde handeln können. Niklas* und ich versicherten ihr, dass ihre mütterliche Intuition auf die Kleine aufpassen würde und sie in Ruhe zu Hause ankommen kann. Auch wenn ich Anna* nach dem ersten Hausbesuch gefestigter wahrnahm, plante ich, in den täglichen Wochenbettbesuchen besonders auf ihre psychische Verfassung zu achten.

In den nächsten Tagen baute Annas* psychischer Zustand merklich ab. Am 7. Tag post partum öffnete sie mir mit tiefen Augenringen und merklich gedrückter Stimmung die Tür. Im Gespräch behauptete sie allerdings, es ginge ihr sehr gut und sie fühle sich sehr wohl in ihrer neuen Rolle als Mutter. Ganz untypisch, da sie sonst jeden Stolperstein ausdiskutiert. Mir war es kaum möglich, mit ihr normal zu kommunizieren. An diesem Tag wollten wir Mia* zum ersten Mal baden. Anna* stand anteilnahmslos daneben und ließ sich auch nach Aufforderung kaum animieren. Ich versuchte, ihr ein paar Tipps für die tägliche Körperpflege zu geben, sie schien mir aber kaum zuzuhören. Sie wirkte desinteressiert, was ich bei ihr nie erlebt hatte. Auf meine Nachfrage, was es bei den beiden zum Mittag gebe, antwortete sie nur: „Nichts, ich habe keinen Hunger.“ Ich suchte nun das Gespräch mit Niklas*. Auch er tat es mit 
dem Schlafmangel durch das Stillen ab und meinte, Anna* komme gut zurecht, auch wenn er in drei Tagen wieder zur Arbeit müsse. Ich bat ihn dennoch, darauf zu achten, dass Anna* regelmäßig isst, und fragte, ob sie viel weine oder unglücklich sei. Niklas* verneinte und führte ihr Verhalten auf die Hormone zurück. Ich fragte ihn, ob er sich an das Thema Wochenbettdepression aus dem Kurs erinnert. Ich sagte, dass sich Anna* m. E. in diesem Zustand befinden könnte. Niklas* versicherte mir, sie sei nur müde und sonst sehr gut drauf. Dennoch versuchte ich, ihn weiter zu sensibilisieren. Er versprach mir, sie gut zu umsorgen, und wir verabredeten uns für den nächsten Tag. Ich fuhr mit sehr gemischten Gefühlen weg und nahm mir vor, am nächsten Tag die EPDS mitzunehmen und mit beiden zu besprechen.

Um 22 Uhr rief mich Niklas* an. Er weinte fast, während er berichtete, dass er inzwischen ebenfalls vermutet, Anna hätte eine Depression. Seit dem Abend habe er das komplette Versorgen von Mia* übernommen. Anna* wolle nicht mal mehr anlegen und lasse die Kleine seit fünf Stunden weinen. Sie wirke, als sei es nicht ihr Kind. Er habe panische Angst, dass Anna* sich und der Kleinen etwas antue. Sie reagiere kaum auf ihn und überhaupt nicht auf Mia*. Während des ganzen Telefonats weinte das Mädchen im Hintergrund. Und trotz der direkten Aufforderung ihres Mannes mochte Anna* Mia* nicht auf den Arm nehmen und stillen. Mit Pre-Nahrung und Flasche fuhr ich zur Familie. Ich versuchte, mit Anna* zu reden, aber auch auf mich reagierte sie abweisend. Sie versuchte halbherzig, ein paar Situationen zu entschuldigen, wirkte aber sehr abwesend. Ich fragte sie, was sie bedrückt oder wie ich ihr helfen kann. Ich bat sie, Mia* an die Brust zu legen, da die Kleine merklich Hunger hatte und die Brust in voller Laktation schon sehr gespannt aussah. Sie verneinte vehement und sagte, sie wolle nur noch Flasche füttern. Das saugende Kind könne sie nicht aushalten.

Ich erklärte Niklas* und Anna*, dass ich die Situation als akute PPD einschätze und dass Anna* sofortige Hilfe benötige. Diese würde sie zunächst in der Frauenklinik bekommen, in der ich sie gern wieder aufnehmen lassen möchte. Mit ihrem Einverständnis rief ich in der Klinik an und klärte die stationäre Aufnahme ab. Meine Sorge war, dass Anna* sich und/oder ihrer Tochter etwas antun könnte. Niklas* stand die Erleichterung ins Gesicht geschrieben. Anna* wirkte nur hilflos. Ich erklärte, warum eine PPD entstehen könne und betonte, dass sie dies nicht beeinflussen kann und dass es nichts über ihre Qualität als Mutter aussagt. Ich wusste, Anna* würde sofort ihre Kompetenz als Mutter infrage stellen. Daher war mir das wichtig.

\section{Der Klinikaufenthalt}

Der diensthabende Arzt nahm Anna* mit Niklas* und Mia* als Begleitpersonen auf. Ich erklärte Anna* die Milchpumpe. Am nächsten Tag bestätigte ein psychiatrisches
Konsil die PPD. Anna* wurde in den nächsten Tagen auf ein stillgeeignetes trizyklisches Antidepressivum eingestellt und es folgten mehrere Akutgespräche mit der Klinikpsychologin. Leider war es nicht möglich, schnell einen Platz in einer Klinik mit Mutter-Kind-Therapie zu bekommen, sodass Anna* nach 10 Tagen wieder nach Hause entlassen wurde. Ihr Zustand war immer noch instabil. Das Desinteresse und die Lethargie waren einer tiefen Traurigkeit und Hilflosigkeit gewichen. Annas* Hausarzt schaffte es, durch Beziehungen einen Termin bei einem Verhaltenspsychologen zu bekommen, zu dem sie dreimal pro Woche ging.

\section{Die Hebammenbetreuung}

Niklas* ließ sich sofort nach Diagnosestellung beurlauben, um für Anna* und Mia* da zu sein. Da Anna* psychisch noch nicht stabil war, hielten alle beteiligten Professionen neben der regelmäßigen Psychotherapie eine regelmäßige Hebammenbetreuung für sehr wichtig. Der behandelnde Gynäkologe stellte unkompliziert ein Rezept für eine weitere Hebammenbetreuung aus. Ich fuhr zunächst weiterhin täglich zu Anna*. Es war ersichtlich, dass sie Unterstützung benötigte, um ihre eigenen Bedürfnisse und die ihres Kindes wahrzunehmen und zu realisieren. In Absprache mit dem behandelnden Psychotherapeuten erstellte ich mit Anna* bei den Hausbesuchen einen Ablaufplan für die nächsten 24 Stunden in Bezug auf die Versorgung ihres Kindes und die Erfüllung ihrer Grundbedürfnisse. Wir fixierten den Plan schriftlich. Das half Anna*, sich an der Struktur zu orientieren. Nach und nach traute sich Anna* wieder zu, Mia* anzulegen und konnte die Nähe immer besser tolerieren. Um die Bindung weiter zu stärken, empfahl ich Anna*, mit Mia* zu baden (Bondingbad) und viel mit nackter Haut zu kuscheln, was sie gern annahm. Nach ca. einem Monat war Anna* so stabil, dass sie körperliche Nähe zu Mia* genoss und sich auch zutraute, allein einen Spaziergang mit Mia* in der Trage zu machen. Es gelang ihr zunehmend besser, den Tag zu strukturieren. So vergrößerten wir die Zeiten zwischen den Hausbesuchen. Auch für die Verhaltenstherapie benötigte sie nur noch zwei Termine pro Woche. Niklas* fing nach zwei Monaten wieder an, drei Tage pro Woche zu arbeiten. Die ersten Wochen schlief Annas* Mutter bei Mia* und ihr, um Anna* in allen Belangen zu unterstützen. Nach drei Monaten konnte Anna* die Nächte allein verbringen. Ich fuhr dann noch einmal wöchentlich zum Hausbesuch. Und sie begann in der Praxis den Rückbildungskurs. Anna* genoss es merklich, wieder unter Menschen zu kommen.

\section{Die Rehabilitation}

Über den Hausarzt beantragte Anna* eine psychosomatische Kur beim Rententräger, zu der sie ihr Kind als Begleitperson mitnehmen und betreuen lassen konnte. Diese wurde schnell genehmigt und sie trat die Kur im Anschluss an den Rückbildungskurs an. Der Kurort lag ganz in der Nähe von Niklas* Arbeitsplatz. Anna* konnte 
dort in Ruhe Kraftreserven aufbauen. Nach neun Monaten wurden die Antidepressiva kontinuierlich reduziert und Anna* war beschwerdefrei und arbeitsfähig. Die ambulante Verhaltenstherapie führte sie noch bis 1,5 Jahre nach der Geburt weiter. Das Paar ist zurzeit erneut in freudiger Erwartung. Als die Schwangerschaft feststand, begann Anna* sich psychologisch unterstützen zu lassen, damit im Falle eines erneuten Auftretens einer PPD sofort reagiert werden kann. Die Beziehung zu ihrem Kind beschreiben die Eltern als glücklich und unkompliziert.

\section{FAZIT}

\section{Reflektion der Hebammenbetreuung}

Die Betreuung von Anna* und ihrer Familie habe ich oft als emotional anstrengend erlebt. Wenn ich dies gespürt habe, war es mir wichtig, mich mit meinen Kollegen / Kolleginnen in der Hebammenpraxis und in der Klinik auszutauschen, um durch verschiedene Perspektiven meine Vorgehensweise zu reflektieren und mir immer wieder meinen Auftrag und meine Grenzen bewusst zu machen. Optimal wäre aus meiner Sicht für Anna* eine stationäre Therapie in einer Klinik mit Mutter-Kind-Therapie gewesen. Da diese Möglichkeit nicht bestand, haben wir gemeinsam interdisziplinär vor Ort nach einem individuellen Weg gesucht, der zum Erfolg führte. Rückblickend ließe sich gewiss die Frage stellen, ob sich durch den Einsatz der EPDS schon in der Schwangerschaft Handlungsbedarf zur Hinzuziehung eines / - r Facharztes / Fachärztin für Psychiatrie oder eines / -er Psychologen / -in ergeben hätte. Dies habe ich in der realen Betreuungssituation jedoch noch nicht als notwendig empfunden.

\section{Autorinnen / Autoren}

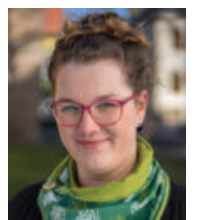

Anne Wiecker arbeitet langjährig als freiberufliche Hebamme mit Vorsorge, Geburtsvorbereitung, Beleggeburten und Wochenbettbetreuung und angestellte Hebamme in Teilzeit in Wernigerode
E-Mail: mail@hebamme-anne-wiecker.de

Literatur

[1] Altmann C. Das Unternehmen Hebamme - Professionalisierung eigener Arbeitsstrukturen innerhalb der Betreuung. Hipp-Hebammenakademie 2016, Fachmagazin Rundherum, Sonderausgabe zum Hipp-Hebammenpreis

[2] Bund Deutscher Hebammen ( $\mathrm{Hg}$.). Psychologie und Psychopathologie für Hebammen - Die Betreuung von Frauen mit psychischen Problemen. Stuttgart, 2006

[3] Knüppel Lauener S, Imhof L. Depression nach der Geburt Screening und Assessment. Die Hebamme 2016; 29: 110-116

[4] Luegmair K. Auswirkungen der postpartalen Depression von Frau und Mann auf die Elternschaft. Die Hebamme 2016; 29: 241-246

[5] Meissner BR. Emotionale Narben aus Schwangerschaft und Geburt auflösen: Mutter-Kind-Bindungen heilen oder unterstützen - in jedem Alter. Meisner Brigitte, 2011

[6] Renz-Polster H. Kinder verstehen. Born to be wild: Wie die Evolution unsere Kinder prägt. Kösel, 2015

[7] Salis B. Psychische Störungen im Wochenbett - Möglichkeiten der Hebammenkunst. Mabuse, 2016

[8] Wortmann-Fleischer S, Downing G, Hornstein C et al. Postpartale psychische Störungen - ein interaktionszentrierter Therapieleitfaden. Kohlhammer, 2016

[9] Wortmann-Fleischer S, Downing G, v. Einsiedel R. Stationäre Eltern-Kind-Behandlung.Stuttgart, 2012

\section{Bibliografie}

DOI https://doi.org/10.1055/a-0893-8642

Die Hebamme 2019; 32: 61-69

(c) Georg Thieme Verlag KG Stuttgart · New York ISSN 0932-8122 